\title{
Inpatient Thromboprophylaxis Use in U.S. Hospitals: Adherence to the Seventh American College of Chest Physician's Recommendations for At-risk Medical and Surgical Patients
}

\author{
Alpesh N. Amin, MD, MBA ${ }^{1}$ \\ Stephen Stemkowski, \\ мHA $^{2}$ \\ Jay Lin, PhD, MBA ${ }^{3}$ \\ Guiping Yang, $\mathrm{ms}^{2}$
}

\author{
${ }^{1}$ An Amin: Department of Medicine, School of Medicine, University of California-Irvine, Orange, California. \\ ${ }^{2}$ S. Stemkowski and G Yang: Premier Research Service, Premier, Inc., Charlotte, North Carolina. \\ ${ }^{3}$ J Lin: Health Outcomes sanofi-aventis, Bridgewater, New Jersey.
}

BACKGROUND: The clinical venous thromboembolism (VTE) burden remains high in the United States, despite guidelines recommending that safe and effective VTE prophylaxis be available. This study assesses the real-world rate of appropriate inpatient VTE prophylaxis in hospitalized U.S. medical and surgical patients at risk of VTE, in accordance with the seventh American College of Chest Physicians, (ACCP) guidelines.

METHODS: Medical and surgical discharges from Premier's Perspective ${ }^{\mathrm{TM}}$ database between January 1, 2005 and December 31, 2006 were considered. Discharges aged $\geq 40$ years, with a length of stay $\geq 6$ days, at risk of VTE due to the presence of $\geq 1$ VTE risk factors identified by the seventh ACCP guidelines, and without contraindications for anticoagulation, were included in the analysis. Appropriate prophylaxis was determined by comparing the daily use, dosage, and duration of anticoagulants and compression devices with the seventh ACCP recommendations for each medical condition or surgical procedure.

RESULTS: A total of 390,024 discharges met the inclusion criteria, of which 201,224 (51.6\%) were medical discharges and $188,800(48.4 \%)$ were surgical discharges. Overall, $65.9 \%$ of medical discharges and $77.7 \%$ of surgical discharges received at least 1 order for VTE prophylaxis during hospitalization. However, only $12.7 \%$ of medical discharges and $16.4 \%$ of surgical discharges received appropriate prophylaxis when the recommended prophylaxis type, dose, and duration from the seventh ACCP guidelines were taken into account.

CONCLUSIONS: Few medical and surgical patients at high risk of VTE receive appropriate inpatient prophylaxis in accordance with guideline recommendations. It is important for individual hospitals to improve VTE prophylaxis practices to meet national performance initiatives. Journal of Hospital Medicine 2009;4:E15-E21. @ 2009 Society of Hospital Medicine.

KEYWORDS: guidelines, thromboprophylaxis, venous thromboembolism.

Venous thromboembolism (VTE) is the third most prevalent cardiovascular disease in the United States, only surpassed by myocardial infarction and stroke. ${ }^{1}$ There are an estimated 600,000 symptomatic VTE events and 300,000 VTE-related deaths per year in the United States, with two-thirds of VTE events being acquired in-hospital. ${ }^{2}$

High rates of VTE remain despite evidence from clinical trials showing that VTE can be safely and effectively reduced by VTE prophylaxis in at-risk medical and surgical patients. ${ }^{1,3,4}$ A cohort study in 2001 suggested that as many as 1 in 6 VTE cases could have been prevented by adequate VTE prophylaxis, amounting to approximately 100,000 preventable cases per year in the United States. ${ }^{5}$ Clinical guidelines are available to guide the practitioner in the choice of prophylaxis regimen and provide evidence-based recommendations on the choice of prophylaxis for each risk-category group. ${ }^{1,6}$

Awareness of the importance of preventing VTE is growing in the United States. The improvement of VTE prevention and VTE treatment has been identified as a key goal for hospitals in the United States by The Joint Commission and the National Quality Forum. ${ }^{7,8}$ Furthermore, a 2006 U.S. Sur- geon General's workshop discussed the issues surrounding deep-vein thrombosis (DVT) prevention, and released a summary of the priority areas for action. ${ }^{9}$ The Surgical Care Improvement Project (SCIP), a national partnership initiative for improving surgical care, has also recently released 2 VTE-focused initiatives (SCIP VTE-1 and SCIP VTE-2) to help reduce preventable causes of mortality and morbidity in surgical patients. ${ }^{10}$

Such quality-assurance initiatives are likely related to the body of data on inadequate use of VTE prophylaxis in U.S. hospitals, including a number of cohort and registry studies that demonstrated low prophylaxis use compared with evidence-based guideline recommendations. ${ }^{5,11-15}$ Although these studies provide an insight into the low levels of use of VTE prophylaxis, recent studies have suggested that the rates of appropriate VTE prophylaxis are even lower when one defines appropriate prophylaxis to include the type or choice of VTE prophylaxis (pharmacological and/or mechanical), drug dose, and duration of prophylaxis. ${ }^{16,17}$

In these real-world studies of U.S. hospital prophylaxis practices from 2002 to $2005,61.8 \%$ of medical patients and 
$72.9 \%$ of surgical patients received at least 1 dose of prophylaxis agent, but only $33.9 \%$ of medical patients and $32.3 \%$ of surgical patients received an appropriate VTE prophylaxis course. $^{16,17}$ These data suggest that the lack of appropriate prophylaxis is not only due to physicians not being aware of the need for VTE prophylaxis, but also due to a lack of understanding of the guideline recommendations.

The seventh update of the American College of Chest Physicians (ACCP) guidelines, released in September 2004, provides more specific recommendations than in previous guidelines. With this publication, and the recent efforts to encourage greater awareness and attention to VTE prophylaxis practices, it is important to see if the combination of increased awareness activities and more specific recommendations have led to an increase in appropriate prophylaxis use compared with previous studies that assessed appropriate prophylaxis with the sixth ACCP guidelines. ${ }^{16,17}$ This study will therefore build on studies from 2001 to 2004 to compare appropriate inpatient prophylaxis use, in accordance with the seventh ACCP guidelines, in a wide range of U.S. medical and surgical patients from January 2005 to December 2006.

\section{Materials and Methods}

\section{Data Source}

The Premier Perspective ${ }^{\mathrm{TM}}$ database is a patient-level dataset of administrative, billing, and discharge information used for comparative analysis of clinical performance. This database contains approximately 5.5 million patient discharges per year from nearly 500 not-for-profit, nongovernmental, community, and teaching hospitals and health systems. Hospitals submit data to the Premier Perspective ${ }^{\mathrm{TM}}$ database on a monthly basis, with the data undergoing numerous quality and validation checks on entry.

\section{Data Collection}

All patient records used in this study were di-dentified in compliance with the Health Insurance Portability and Accountability Act (HIPAA) of 1996 (http://www.hhs.gov/ ocr/hipaa). Hospital and patient demographics, discharge information, principal and secondary diagnoses and procedures, and detailed resource consumption information for each discharge by day of hospitalization were extracted from the Premier Perspective ${ }^{\mathrm{TM}}$ database. Records related to the same hospital discharge were linked using a nonpersonal identifier assigned by the provider that prevented subject identification and the linking of identifiers to subjects. Since this study did not involve "identifiable human subjects," it was exempt from Institutional Review Board overview under the Common Rule (45 CFR \$46.101(b)(4)). ${ }^{18}$

\section{Patient Discharge Selection Criteria}

The selected study population of patients at high risk of VTE was derived from discharge records of patients discharged between January 1, 2005 and December 31, 2006 that met all inclusion criteria. Data were obtained from 429 hospitals across 39 states in the United States that submitted detailed patient hospitalization information by day of stay in monthly reports to the Premier Perspective ${ }^{\mathrm{TM}}$ database.

Inclusion criteria were:

$1 \geq 40$ years old.

2. Minimum hospital length of stay of 6 days (based on the inclusion criteria from the Prophylaxis in Medical Patients with Enoxaparin (MEDENOX) trial, which first demonstrated a reduction in 14-day VTE rates in medical patients receiving low-molecular weight heparin (LMWH) compared with placebo. ${ }^{3}$

3. Deemed at-risk of VTE due to the presence of 1 or more of the VTE risk factors identified by the seventh ACCP guidelines. ${ }^{1}$

4. Principal medical diagnosis or surgical procedure (based on the International Classification of Diseases, ninth Revision, Clinical Modification [ICD-9 CM] coding system) belonging to an acute medical illness or a surgical procedure group. Medical diagnoses were as follows: heart failure, burns, severe lung disease, cancer (with or without surgery), acute spinal cord injury (without surgery), and trauma (without surgery). Surgical procedure groups were as follows: major orthopedic surgery, general surgery, gynecological surgery, laparoscopic surgery, urological surgery, and neurological surgery. A principal diagnosis or procedure code was assigned to each patient by the hospital. Where multiple surgeries were performed, the principal procedure assigned to the discharge was used. Discharges with both a principal medical diagnosis and a principal surgical procedure were excluded to eliminate uncertainty about which ACCP guideline recommendation should be applied, except in the cancer group where prophylaxis recommendations for a surgical procedure took priority if present. A final group, the critical care group, was also studied. The critical care group consisted of any discharge from the above medical and surgical groups that was flagged for the critical care unit. Appropriate prophylaxis in this group was defined by their principal medical or surgical diagnosis.

5. Absence of any contraindication that required-modification to ACCP-recommended anticoagulant therapy. Patient discharges were excluded if they had ICD-9 CM codes for active peptic ulcer disease, malignant hypertension, blood disease (iron deficiency and other anemias, hereditary hemolytic anemias, hereditary elliptocytosis, anemias due to disorders of glutathione metabolism, thalassemias, sickle-cell trait and disease, other hemoglobinopathies, acquired hemolytic anemias, aplastic and other unspecified anemias, coagulation defects, purpura, and other hemorrhagic conditions), human immunodeficiency virus (HIV) infection, pregnancy, VTE present on admission, intubations of the 
gastrointestinal and respiratory tracts, liver disease, thrombocytopenia, or insufficient renal function (severe or moderate renal insufficiency). ${ }^{19}$

\section{Any and Appropriate VTE Prophylaxis Definitions}

The use of guideline-recommended pharmacological prophylaxis (unfractionated heparin, enoxaparin, dalteparin, tinzaparin, fondaparinux, or warfarin) or mechanical prophylaxis (intermittent pneumatic compression or elastic stockings) was collected and measured for each patient discharge included in the study.

Any prophylaxis was defined as the discharge receiving at least 1 order for pharmacological or mechanical prophylaxis during the hospital stay. Appropriate prophylaxis was defined as the discharge receiving VTE prophylaxis that was in accordance with the recommendations for that discharge's principal diagnosis in the seventh ACCP guidelines. ${ }^{1}$ In order for a discharge to have received appropriate prophylaxis in this study, a detailed examination of the hospital administrative records had to show that the discharge received a guideline-recommended VTE prophylaxis regimen (pharmacological or mechanical) at the appropriate dose (if a pharmacological regimen was recommended) and for the appropriate duration. The regimens that were derived from the seventh ACCP guidelines and considered as appropriate prophylaxis in this study can be seen in Appendix A. All VTE prophylaxis had to be provided daily for the length of the discharge's hospital stay minus 2 days. The allowance for 2 missing days was to accommodate for the possibility of partial days of stay occurring at admission and discharge, or for the possibility of an invasive procedure occurring during hospitalization for which anticoagulation is not recommended on the day of the procedure. Due to the short hospital length of stay in orthopedic surgery discharges, the duration of prophylaxis had to reach a minimum of length of stay minus 2 days or 7 days total in order to be deemed appropriate.

Levels of any and appropriate prophylaxis were compared between the medical and surgical discharge groups. Furthermore, the influence of factors that may have affected the levels of appropriate prophylaxis such as admission source, geographical region, and hospital type, size, and location was studied. In discharges where VTE prophylaxis did not meet the criteria for appropriate prophylaxis, the reasons were collected and compared between discharge groups. Potential reasons for not receiving appropriate prophylaxis were receiving no pharmacological prophylaxis when prophylaxis was recommended, receiving mechanical prophylaxis alone when pharmacological prophylaxis was recommended, receiving an insufficient dose of pharmacological prophylaxis, or receiving an insufficient duration of prophylaxis.

\section{Results}

Among the 2,353,287 discharges in the database during the study period, 390,024 (16.6\%) discharges were included in

\begin{tabular}{ll} 
TABLE 1. Study Populations for Acute & Medical and Major \\
Surgical IIInesses & Number of Discharges \\
\hline Diagnostic Group & \\
\hline & \\
Medical Groups & 229 \\
Acute spinal cord injury & 973 \\
Burns & 57,792 \\
Cancer & 21,119 \\
Trauma & 34,286 \\
Heart failure & 86,825 \\
Severe lung disease & 97,022 \\
Critical care* & 201,224 \\
Total medical & \\
Surgical Groups & 61,157 \\
General surgery & 601 \\
Gynecological surgery & 23,341 \\
Laparoscopic surgery & 4021 \\
Major orthopedic surgery & 1071 \\
Elective hip arthroplasty & 2616 \\
Elective knee arthroplasty & 13 \\
Emergency knee arthroplasty & 51 \\
Hip fracture surgery & 270 \\
Elective spinal surgery & 4142 \\
Urological surgery & 4811 \\
Neurological surgery & 90,727 \\
Vascular surgery & 188,800 \\
Total surgical & \\
\hline & *The critical care group comprises discharges from all other groups that in addition were flagged for \\
the critical care unit. These discharges are therefore already accounted for in the numbers of their pri- \\
mary diagnosis group. \\
\hline
\end{tabular}

this analysis. Of these discharges, 201,224 (51.6\%) were in acute medical illness groups and $188,800(48.4 \%)$ were in surgical procedure groups (Table 1). The medical and surgical groups containing the highest numbers of discharges were critical care (97,022 discharges) and vascular surgery (90,727 discharges), respectively (Table 1 ).

The total rate of any prophylaxis in this analysis was $71.6 \%$, meaning that nearly 3 in every 4 discharges that were eligible for VTE prophylaxis received at least 1 order for pharmacological or mechanical VTE prophylaxis (Table 2 ). Rates of any prophylaxis were lower for medical discharges at $65.9 \%$, compared with $77.7 \%$ in surgical discharges. Variation was observed within individual discharge diagnosis groups, with the highest rate of any prophylaxis being $93.8 \%$ in the major orthopedic surgery group and the lowest rate being $36.8 \%$ in the burns group (Table 2).

However, when the recommendations of the seventh ACCP guidelines were applied for prophylaxis type, dose, and duration, only $14.5 \%$ of all patients received appropriate prophylaxis (Table 2). Medical discharges also received lower levels of appropriate prophylaxis at $12.7 \%$ than surgical discharges (16.4\%). Large variations in the rates of appropriate prophylaxis were observed between discharge groups in both the medical and surgical populations. In the medical groups, the highest rate of appropriate prophylaxis was $17.5 \%$ in trauma discharges, and the lowest rate was 
TABLE 2. Aggregate Any Prophylaxis and Appropriate Prophylaxis Rates by Medical or Surgical Discharge Category

\begin{tabular}{lll} 
Discharge Group & Any Prophylaxis (\%) & Appropriate Prophylaxis (\%) \\
\hline Medical groups & 65.9 & 12.7 \\
Acute spinal injury & 81.2 & 10.0 \\
Burns & 36.8 & 4.7 \\
Cancer & 69.4 & 12.5 \\
Trauma & 69.4 & 17.5 \\
Heart failure & 79.8 & 15.9 \\
Severe lung disease & 51.8 & 10.5 \\
Surgical groups & 77.7 & 16.4 \\
General & 66.4 & 13.3 \\
Gynecological & 89.7 & 7.7 \\
Laparoscopic & 79.5 & 11.3 \\
Orthopedic & 93.8 & 48.6 \\
Urological & 66.8 & 6.3 \\
Neurological & 69.8 & 5.7 \\
Vascular & 85.0 & 19.5 \\
Critical care* & 89.9 & 15.7 \\
Total & 71.6 & 14.5 \\
\hline
\end{tabular}

* The critical care group comprises discharges from all other groups that in addition were flagged for the critical care unit. Appropriate prophylaxis was therefore defined as the prophylaxis appropriate for the discharge primary medical diagnosis or surgical procedure.

$4.7 \%$ in burns discharges. In the surgical groups, the highest rate of appropriate prophylaxis was $48.6 \%$ in major orthopedic surgery discharges, and the lowest rate was $5.7 \%$ in neurological surgery discharges.

Further examination of the individual discharge records reveals that the primary reason that discharges in the medical diagnosis groups did not receive appropriate prophylaxis was due to no pharmacological prophylaxis being provided, despite the lack of a contraindication to anticoagulant therapy (Table 3). A total of $34.1 \%$ of all medical discharges received no pharmacological prophylaxis when indicated. Other reasons for medical discharges not receiving appropriate prophylaxis were receiving pharmacological prophylaxis at an incorrect dose (lower than the guideline-recommended daily total; $22.7 \%$ ), receiving prophylaxis for an insufficient duration (missing at least 1 day of prophylaxis that was not the admission or discharge date; $22.1 \%$ ), and receiving mechanical prophylaxis alone when pharmacological prophylaxis was recommended $(8.4 \%)$. Variation in the reasons for not receiving appropriate prophylaxis was observed between medical diagnosis groups, with the primary reason being mechanical prophylaxis alone in acute spinal injury discharges, and no prophylaxis in burns, cancer, and trauma discharges (Table 3).

In the total surgical discharge population, an insufficient duration of prophylaxis was the main reason for not receiving appropriate prophylaxis, with $36.1 \%$ of all surgical discharges receiving an insufficient duration of prophylaxis (Table 3). Other reasons for surgical discharges not receiving appropriate prophylaxis were receiving no pharmacological prophylaxis (22.3\%), receiving pharmacological prophylaxis at an incorrect

\section{TABLE 3. Rates and Reasons of Inappropriate Prophylaxis Within the Entire Study Population per Discharge Group}

\begin{tabular}{|c|c|c|c|c|}
\hline & $\begin{array}{l}\text { Inappropriate } \\
\text { Dose (\%) }\end{array}$ & $\begin{array}{l}\text { Insufficient } \\
\text { Duration (\%) }\end{array}$ & $\begin{array}{l}\text { Mechanical } \\
\text { Prophylaxis } \\
\text { Only (\%) }\end{array}$ & $\begin{array}{l}\text { No prophylaxis } \\
\text { Ordered (\%) }\end{array}$ \\
\hline Medical groups & 22.7 & 22.1 & 8.4 & 34.1 \\
\hline Acute spinal injury & 15.3 & 26.2 & 29.7 & 18.8 \\
\hline Burns* & 14.9 & 12.0 & 5.1 & 63.2 \\
\hline Cancer & 18.3 & 22.3 & 16.3 & 30.6 \\
\hline Trauma* & 12.6 & 19.7 & 19.6 & 30.6 \\
\hline Heart failure & 22.1 & 39.3 & 1.6 & 21.1 \\
\hline Severe lung disease & 19.5 & 17.5 & 3.0 & 50.0 \\
\hline Surgical groups & 13.7 & 36.1 & 11.5 & 22.3 \\
\hline General & 10.9 & 24.5 & 17.6 & 33.6 \\
\hline Gynecological & 11.8 & 23.6 & 46.6 & 10.3 \\
\hline Laparoscopic & 21.4 & 19.7 & 27.1 & 20.5 \\
\hline Orthopedic & 39.8 & 1.6 & 3.7 & 6.2 \\
\hline Urological & 12.6 & 24.8 & 23.0 & 33.2 \\
\hline Neurological & 15.2 & 16.2 & 32.7 & 30.2 \\
\hline Vascular & 12.4 & 51.2 & 1.9 & 15.0 \\
\hline Critical care $^{\dagger}$ & 14.2 & 49.4 & 10.5 & 10.1 \\
\hline \multicolumn{5}{|c|}{$\begin{array}{l}\text { *The trauma and burns groups contains only discharges that did not have surgery. } \\
\text { 'The critical care group comprises discharges from all other groups that in addition were flagged for } \\
\text { the critical care unit. Appropriate prophylaxis was therefore defined as the prophylaxis appropriate for } \\
\text { the discharge primary medical diagnosis or surgical procedure. }\end{array}$} \\
\hline
\end{tabular}

dose (13.7\%), and receiving mechanical prophylaxis alone $(11.5 \%)$. Variation in the reasons for not receiving appropriate prophylaxis was also observed between surgical diagnosis groups, with the primary reason being inappropriate duration in vascular surgery discharges, mechanical prophylaxis alone in gynecological, laparoscopic, and neurological surgery discharges, inappropriate dosage in orthopedic surgery discharges, and no prophylaxis provided in general and urological surgery discharges (Table 3). In medical and surgical discharges that had a critical care unit stay during their hospitalization, only $15.7 \%$ received appropriate prophylaxis, with nearly one-half of all critical care discharges receiving an insufficient duration of VTE prophylaxis (Table 3).

Analysis of the mean rates of appropriate prophylaxis by hospital factors suggests that trends exist toward increased use of appropriate prophylaxis in larger hospitals, in urban hospitals compared with rural hospitals, and in teaching compared with nonteaching hospitals (Table 4).

\section{Discussion}

This study suggests that appropriate prophylaxis, as defined in current practice guidelines for the prevention of VTE in specific at-risk groups, is not widely applied in a selected cohort of hospitalized patients with known risks for VTE. Current ACCP guidelines provide specific direction on safe and effective prophylaxis regimens. These recommendations include the appropriate dosing and appropriate duration of prophylaxis, according to the specific risk in defined medical and surgical risk groups. However, in nearly 400,000 


\begin{tabular}{|c|c|c|}
\hline & $\begin{array}{l}\text { Medical } \\
\text { Discharges (\%) }\end{array}$ & $\begin{array}{l}\text { Surgical } \\
\text { Discharges (\%) }\end{array}$ \\
\hline \multicolumn{3}{|c|}{ Hospital size (number of beds) } \\
\hline $0-99$ & 7.5 & 11.0 \\
\hline $100-299$ & 9.6 & 13.4 \\
\hline $300-499$ & 13.1 & 16.2 \\
\hline $500+$ & 14.9 & 18.5 \\
\hline \multicolumn{3}{|l|}{ Teaching status } \\
\hline Teaching & 16.5 & 18.9 \\
\hline Nonteaching & 9.9 & 14.2 \\
\hline \multicolumn{3}{|l|}{ Location } \\
\hline Urban & 13.2 & 16.7 \\
\hline Rural & 8.9 & 14.0 \\
\hline \multicolumn{3}{|l|}{ Admission source } \\
\hline Emergency department & 12.4 & 15.4 \\
\hline Physician referral & 12.2 & 17.2 \\
\hline Other & 22.2 & 19.9 \\
\hline \multicolumn{3}{|l|}{ Primary payor } \\
\hline Commercial & 13.3 & 16.5 \\
\hline Managed care & 13.6 & 16.8 \\
\hline Medicaid & 11.7 & 12.7 \\
\hline Medicare & 12.3 & 17.0 \\
\hline Other payors & 13.7 & 15.6 \\
\hline \multicolumn{3}{|l|}{ Geographical region } \\
\hline East North Central & 19.2 & 25.1 \\
\hline East South Central & 8.3 & 14.0 \\
\hline Middle Atlantic & 20.3 & 21.1 \\
\hline Mountain & 15.7 & 19.4 \\
\hline New England & 10.9 & 12.3 \\
\hline Pacific & 11.0 & 11.7 \\
\hline South Atlantic & 10.5 & 14.3 \\
\hline West North Central & 7.5 & 13.2 \\
\hline West South Central & 8.9 & 15.0 \\
\hline
\end{tabular}

medical and surgical discharges at risk for VTE in U.S. hospitals, only $14.5 \%$ of discharges received VTE prophylaxis that met the recommendations of the seventh ACCP guidelines for prophylaxis type, dose, and duration. Although $71.6 \%$ of discharges received some form of prophylaxis during hospitalization, the majority of these discharges did not receive "appropriate prophylaxis." Furthermore, nearly $30 \%$ of patients who should have received prophylaxis did not have a single order for prophylaxis during their hospitalization.

The ACCP has regularly updated its VTE prevention guidelines from the first release of the guidelines in 1986 to the most recent seventh guidelines in $2008 .^{20,21}$ These updates have been in line with emerging literature for both patient populations that are at risk for VTE, and for VTE prophylaxis regimens that are safe and effective in these patients. The main changes between the two most recent guidelines in VTE prevention (sixth and seventh) were to introduce risk assessment within patient groups, resulting in a greater number of more stringent recommendations. ${ }^{1,11}$
The combination of the more stringent recommendations, and the recently growing national focus on the need for improved VTE prevention from groups such as The Joint Commission and the National Quality Forum would suggest that the levels of appropriate VTE prophylaxis in U.S. hospitals should be increasing. ${ }^{7,8}$

The number of patients, including both medical and surgical discharges, in this study that were eligible for prophylaxis was approximately $16.6 \%$. This number is substantially lower than previously reported in a recent U.S. study that found that $31 \%$ of U.S. hospital discharges in 2003 were at risk of VTE. ${ }^{22}$ It is likely that the discrepancy between the 2 studies is due to the more stringent length of stay criteria ( $\geq 6$ days) in our study compared to $\geq 2$ days in the Anderson et al. $^{22}$ study. This length of stay criteria will have likely selected for complicated, higher-risk patients and as such the results of this study may be more applicable to patients at higher risk of VTE than to the general population.

However, when the results of this study are compared to similar studies of appropriate prophylaxis with the sixth ACCP guidelines during the period of 2002 to 2005, the level of appropriate prophylaxis appears to have decreased. ${ }^{16,17}$ Although strong conclusions can not be drawn from the comparison of the analyses, the appropriate prophylaxis in medical patients during the timeframe of the sixth ACCP guidelines occurred in $33.9 \%$ of patients, compared with only $13.7 \%$ in the present study. Two of the categories with the highest rates of appropriate VTE prophylaxis in the analysis of the sixth ACCP guidelines were not included in our study (acute myocardial infarction and ischemic strokedue to these patients being likely to receive treatment dose anticoagulants), but the categories that were included in both studies, ie, acute spinal cord injury, cancer, heart failure, and severe lung disease, have a $50 \%$ to $66 \%$ decrease in appropriate prophylaxis rates in the current study. ${ }^{16}$ Only trauma patients have similar rates between studies. Interestingly, the rates of any prophylaxis have increased in the current study, with $65.9 \%$ of medical patients receiving some form of prophylaxis in this analysis, compared with $61.8 \%$ in the prior study. Similar results are observed when comparing the surgical population in this analysis to prior data on surgical discharges, with the rate of any prophylaxis being higher with the seventh ACCP guidelines than the sixth ACCP guidelines $(77.7 \%$ vs. $72.9 \%$, respectively), but the rate of appropriate prophylaxis being lower (16.4\% vs. $32.3 \%$, respectively). ${ }^{17}$

The combination of an increase in any prophylaxis, but a decrease in appropriate prophylaxis may suggest that the overall national awareness of the need for VTE prophylaxis in at-risk patients is increasing. However, the combination of more stringent guideline recommendations, and perhaps a lack of awareness as to the guideline recommendations themselves, has actually led to a decrease in the amount of appropriate prophylaxis being prescribed. Despite this, there still remain approximately $30 \%$ of patients who receive no prophylaxis at all. To this end, it is important that awareness 
initiatives and quality improvement programs address both the need for prophylaxis, and the most safe and effective way to provide appropriate prophylaxis in specific patient populations. The use of electronic or manual alerts and order forms for VTE prophylaxis is one effective way of increasing appropriate prophylaxis, and ultimately reducing the incidence of hospital-acquired VTE. ${ }^{23}$ A pivotal study by Kucher et al. $^{23}$ studied over 2500 patients who were randomly assigned to an electronic intervention group or a control group. In the intervention group, the physician received an electronic alert of the patients' VTE risk, whereas in the control group no alert was issued. The study found that, compared to control, both pharmacological prophylaxis $(23.6 \%$ vs. $13.0 \%, P<0.001)$ and mechanical prophylaxis $(10.0 \%$ vs. $1.5 \%, P<0.001)$ were prescribed more frequently in the intervention group. Furthermore, this led to a significant reduction in the incidence of clinically diagnosed, objectively confirmed deep-vein thrombosis or pulmonary embolism at 90 days, with an incidence of $4.9 \%$ in the intervention group compared with $8.2 \%$ in the control group ( $P<0.001$ ). As this study has found that prophylaxis is inappropriately provided due to insufficient prescribing, insufficient duration, and inappropriate dosing, it would be interesting to identify the educational or procedural interventions that have the biggest impact on each factor. This would allow hospitals to create multicomponent initiatives with a greater chance of increasing the rates of appropriate prophylaxis.

A strength of this study is that this is the largest database analysis of hospital discharges and seventh ACCP guidelinerecommended VTE prophylaxis use to date, giving insights into real-world clinical practice in the United States with the most recent guidelines. This will provide a checkpoint for improvements in advance of the 2008 guidelines being released. A limitation of this study is that we have utilized a conservative approach to selecting patients who were clearly at risk of VTE. Patients were required to have a length of stay $\geq 6$ days. This may have both excluded a number of orthopedic surgery and medical patients despite their requirements for VTE prophylaxis and likely have selected a cohort of sicker patients at high-risk for VTE. It is possible that this will have created a bias for specific patient or hospital characteristics (eg, complex patients or hospitals with less efficient systems) that we cannot adjust for, and this may have affected the results of the study. Due to the use of hospital records alone, we are also unable to examine whether discharges continued to receive appropriate prophylaxis following discharge. As some orthopedic surgery patients are recommended to receive prophylaxis for up to 28 to 35 days following surgery, ${ }^{1}$ this limitation is likely to have resulted in an overestimation of appropriate prophylaxis rates in the current study. However, it is important to note that the appropriate prophylaxis rate was extremely low, even in this selected higher-risk population. Furthermore, the use of length of stay minus 2 days as the criteria for appropriate duration may have led to a slight underestimation of appro- priate prophylaxis, especially as the reasons for any interruption of prophylaxis by the physician during the hospital stay are unknown. An additional limitation is that the study uses retrospective discharge record data that cannot fully evaluate whether the prophylaxis was appropriate in a complex individual patient. For example, contraindications to anticoagulant prophylaxis are not always documented and may not have been identified in the hospital coding exclusion criteria. In addition, we are only able to assess whether mechanical prophylaxis was ordered, and not whether it was appropriately used. Another limitation is that basing assignment of prophylaxis on the principal diagnosis increases the likelihood that clinical decisions on prophylaxis were based on the primary reason for admission, when in reality there may have been multiple factors affecting the patient's risk assessment and the physician's prophylaxis decision. In this analysis, we used the ACCP guidelines as these are currently the most long-standing VTE prophylaxis guidelines available, as well as being the most comprehensive for multiple patient groups. However, it is important to acknowledge that specific specialties, such as oncologists and orthopedic surgeons, also have their own specialized guidelines which may have different recommendations. This may therefore have led to an underestimation of appropriate prophylaxis. In addition, the ACCP guidelines have been updated in 2008, providing physicians with a revised set of recommendations for VTE prophylaxis. ${ }^{21}$ We utilized the 2004 guidelines in our analysis as we feel that it is important to assess whether the prophylaxis provided was appropriate by the standards of care during the timeframe within which the data were collected. However, we acknowledge that applying the new guidelines may impact the results of the study. One final consideration that would make an interesting follow-up study is an assessment of whether appropriate or inappropriate prophylaxis impacts the clinical outcomes. For example, do patients with appropriate prophylaxis have fewer VTE events and improved mortality compared with those without prophylaxis or with inappropriate prophylaxis.

In summary, this work identifies that, in the United States, there is still considerable underutilization of appropriate VTE prophylaxis across a broad range of diagnostic groups with known VTE risk. While nearly three-quarters of patients do receive at least 1 order for VTE prophylaxis during their hospitalization, only approximately 1 in 7 patients receive appropriate prophylaxis that matches evidencebased recommendations for type, dose, and duration. Physician awareness of both the need for VTE prophylaxis, and more specifically what constitutes appropriate prophylaxis in certain patient groups, needs to be increased. The current national performance initiatives will provide a framework for this improvement, but it is the responsibility of individual hospitals to improve their VTE prophylaxis practices. Such an improvement across hospitals will lead to a sizeable reduction in the incidence and economic burden of VTE on the U.S. healthcare system. 


\section{Acknowledgements}

Editorial and financial support for this publication was provided by sanofi-aventis U.S., Inc. The authors, however, are fully responsible for the content and editorial decisions for this work. A.A. is a research consultant and on the speakers bureau for sanofi-aventis U.S., Inc. S.S. and G.Y. work for Premier, Inc., and received funding to carry out this work from sanofi-aventis U.S., Inc. J.L. is an employee of sanofi-aventis U.S., Inc.

\section{Address for correspondence and reprint requests:}

Alpesh N. Amin, MD, MBA, University of California-Irvine, 101

The City Drive South, Building 58, Room 110, ZC-4076H, Orange, CA 92868; Telephone: 714456 3785; Fax: 714456 3875; E-mail: anamin@uci.edu Received 22 May 2008; revision received 4 January 2009; accepted 14 February 2009.

\section{References}

1. Geerts WH, Pineo GF, Heit JA, et al. Prevention of venous thromboembolism: the Seventh ACCP Conference on Antithrombotic and Thrombolytic Therapy. Chest. 2004;126:338S-400S.

2. Heit JA, Cohen AT, Anderson FA. Estimated annual number of incident and recurrent, non-fatal and fatal venous thromboembolism events in the US. Blood. 2005;106:Abstract 910.

3. Samama MM, Cohen AT, Darmon Y-V, et al. A comparison of enoxaparin with placebo for the prevention of venous thromboembolism in acutely ill medical patients. Prophylaxis in Medical Patients with Enoxaparin Study Group. N Engl J Med. 1999;341:793-800.

4. ENOXACAN Study Group. Efficacy and safety of enoxaparin versus unfractionated heparin for prevention of deep vein thrombosis in elective cancer surgery: a double-blind randomized multicentre trial with venographic assessment. ENOXACAN Study Group. Br J Surg. 1997;84:1099-1103.

5. Arnold DM, Kahn SR, Shrier I. Missed opportunities for prevention of venous thromboembolism: an evaluation of the use of thromboprophylaxis guidelines. Chest. 2001;120:1964-1971.

6. Cardiovascular Disease Educational and Research Trust; Cyprus Cardiovascular Disease Educational and Research Trust; European Venous Forum; International Surgical Thrombosis, Forum; International Union of Angiology; Union Internationale de Phlebologie. Prevention and treatment of venous thromboembolism. International Consensus Statement (Guidelines According to Scientific Evidence). Int Angiol. 2006;25:101-161.

7. Chyna JT. Preparing for DVT core measures. Healthcare leaders should begin preparing for new deep vein thrombosis prevention standards. Healthc Exec. 2005;20:66-67.

8. Joint Commission on Accreditation of Healthcare Organizations (JCAHO). Available at: http://www.jointcommission.org. Accessed May 2009.
9. U.S. Surgeon General. Summary and consideration of priority areas for action: Surgeon General's workshop on deep-vein thrombosis. Available at: http://www.surgeongeneral.gov/topics/deepvein/workshop/presentations/summary.pdf. Accessed May 2009.

10. Medicare Quality Improvement Committee. SCIP Project Information. Available at: http://www.qualitynet.org/dcs/ContentServer?c=MQParents \&pagename $=$ Medqic\%2FContent\%2FParentShellTemplate\&cid=11229049 30422\&parentName=Topic Accessed October 2006.

11. Geerts WH, Heit JA, Clagett GP, et al. Prevention of venous thromboembolism. Chest. 2001;119:132S-175S.

12. Vallano A, Arnau JM, Miralda GM, Pérez-Bartolí J. Use of venous thromboprophylaxis and adherence to guideline recommendations: a crosssectional study. Thromb J. 2004;2:3-9.

13. Aujesky D, Guignard E, Pannatier A, Cornuz J. Pharmacological thromboembolic prophylaxis in a medical ward: room for improvement. J Gen Intern Med. 2002;17:788-791.

14. Goldhaber SZ, Tapson VF. A prospective registry of 5,451 patients with ultrasound-confirmed deep vein thrombosis. Am J Cardiol. 2004;93: 259-262.

15. Tapson VF, Hyers TM, Waldo AL, et al. Antithrombotic therapy practices in US hospitals in an era of practice guidelines. Arch Intern Med. 2005; 165:1458-1464.

16. Amin A, Stemkowski S, Lin J, Yang G. Thromboprophylaxis rates in US medical centers: success or failure? J Thromb Haemost. 2007;5:1610-1616.

17. Amin A, Stemkowski SA, Lin J, Yang G. Preventing venous thromboembolism in US hospitals: are surgical patients receiving appropriate prophylaxis? Thromb Haemost. 2008;99:796-797.

18. U.S. Department of Health and Human Services. Policy for Protection of Human Research Subjects. Available at: http://www.hhs. gov/ohrp/humansubjects/guidance/45cfr46.htm\#46.101. Accessed: May 2009 .

19. McGarry LJ, Thompson D. Retrospective database analysis of the prevention of venous thromboembolism with low-molecular-weight heparin in acutely III medical inpatients in community practice. Clin Ther. 2004;26: 419-430.

20. ACCP/NHLBI National Conference on Antithrombotic Therapy. Chest. 1986;89:1S-106S.

21. Geerts WH, Bergqvist D, Pineo GF, et al. Prevention of venous thromboembolism: American College of Chest Physicians Evidence-Based Clinical Practice Guidelines (8th edition). Chest. 2008;133:381S-453S.

22. Anderson A, Zayaruzny M, Heit JA, Fidan D, Cohen AT. Estimated annual numbers of US acute-care hospital patients at risk for venous thromboembolism. Am J Hematol. 2007;82:777-782.

23. Kucher N, Koo S, Quiroz R, et al. Electronic alerts to prevent venous thromboembolism among hospitalized patients. N Engl J Med. 2005;352: 969-977. 